\title{
INTERPOLATION BETWEEN WEIGHTED HARDY SPACES
}

\author{
MICHAEL CWIKEL, JOHN E. MCCARTHY, AND THOMAS H. WOLFF
}

(Communicated by Paul S. Muhly)

\begin{abstract}
We prove that $H^{p}\left(w_{0}^{1-s} w_{1}^{s}\right)$ is an interpolation space of exponent $s$ between $H^{p}\left(w_{0}\right)$ and $H^{p}\left(w_{1}\right)$ if and only if $\log \left(w_{1} / w_{0}\right)$ is in BMO. If $\log \left(w_{1} / w_{0}\right)$ fails to be in $\mathrm{BMO}, H^{p}\left(w_{0}^{1-s} w_{1}^{s}\right)$ can still be an interpolation space, provided the range of $w$ has sufficiently large gaps.
\end{abstract}

\section{INTRODUCTION}

The first theorem on the interpolation of linear operators is due to Riesz [Ri], who showed that if $T$ is a linear operator that is continuous from $L^{p_{0}}(\mu)$ to $L^{p_{0}}(\mu)$ and from $L^{p_{1}}(\mu)$ to $L^{p_{1}}(\mu)$, then it is continuous from $L^{p}(\mu)$ to $L^{p}(\mu)$, for all $1 \leq p_{0} \leq p \leq p_{1} \leq \infty$. Many interpolation theorems have been proved since then; we mention only a result of Stein [St] that allows the measure to change: if $T$ is continuous from $L^{p}\left(w_{0} \mu\right)$ to $L^{p}\left(w_{0} \mu\right)$, and from $L^{p}\left(w_{1} \mu\right)$ to $L^{p}\left(w_{1} \mu\right)$, then it is continuous from $L^{p}\left(w_{0}^{1-s} w_{1}^{s} \mu\right)$ to itself, for every $0 \leq s \leq 1$.

In this paper we consider the question of when linear operators defined and continuous on two weighted Hardy spaces must also be continuous on some other interpolating weighted Hardy space. By a weight we shall always mean a nonnegative measurable function $w$ on the unit circle that satisfies $\int_{\mathrm{T}}|\log (w)| d \sigma<\infty$, where $\sigma$ is a normalised Lebesgue measure. If $w$ is also integrable, the weighted Hardy space $H^{p}(w)$ is the closure in $L^{p}(w \sigma)$ of the (analytic) polynomials, for $1 \leq p<\infty$. This is isometrically isomorphic to the space $\psi H^{p}$, where $H^{p}$ is the classical Hardy space (i.e., $\left.w \equiv 1\right), \psi$ is the outer function with modulus $w^{-1 / p}$, and the norm of $\psi f$ in $\psi H^{p}$ is defined to be the norm of $f$ in $H^{p}$ (see [Ga] for the definition of an outer function). For an arbitrary weight, one can always find such an outer function (because of the log-integrability assumption), and so we define $H^{p}(w)$ to be $\psi H^{p}$. For simplicity, we shall always assume that $p \geq 1$.

Given any two Banach spaces $X_{0}$ and $X_{1}$ that are 'compatible', in the sense of both being continuously embedded in some topological vector space, there are two principal ways of defining a family of intermediate spaces $X_{s}$ such that any linear operator that is continuous from $X_{0}$ to $X_{0}$ and from $X_{1}$ to $X_{1}$ is

Received by the editors September 24, 1990 and, in revised form, February 28, 1991.

1980 Mathematics Subject Classification (1985 Revision). Primary 46M35, 46E15; Secondary 30D55, 47B38. 
also continuous from $X_{s}$ to $X_{s}, 0<s<1$ : these are the so-called real and complex methods (see [BL] for details). Most interpolation theorems are proved by showing that the desired space is isomorphic to one of these $X_{s}$ 's. Both these methods have the property that the norm of an operator $T$ on $X_{s}$ is less than or equal to $\|T\|_{X_{0}}^{1-s}\|T\|_{X_{1}}^{s}$. By analogy with Stein's theorem, one might expect that the space $H^{p}\left(w_{0}^{1-s} w_{1}^{s}\right)$ would be isomorphic to an interpolation space between $H^{p}\left(w_{0}\right)$ and $H^{p}\left(w_{1}\right)$ obtained by one of these methods. In $\S 1$ we prove that this is true if and only if $\log \left(w_{1} / w_{0}\right)$ is in the space BMO of functions of bounded mean oscillation (see [Ga] for a definition of $\mathrm{BMO}$ ). If $\log \left(w_{1} / w_{0}\right)$ is not in BMO, we show that $H^{p}\left(w_{0}^{1-s} w_{1}^{s}\right)$ is not an interpolation space of exponent $s$ (i.e., it does not satisfy $\|T\|_{H^{p}\left(w_{0}^{1-s} w_{1}^{s}\right)} \leq C\|T\|_{H^{p}\left(w_{0}\right)}^{1-s}\|T\|_{H^{p}\left(w_{1}\right)}^{s}$ for all linear operators bounded on both $H^{p}\left(w_{0}\right)$ and $\left.H^{p}\left(w_{1}\right)\right)$.

In $\S 2$ we give an example of a weight $w$ whose logarithm is not in BMO, but such that $H^{2}(\sqrt{w})$ is an interpolation space between $H^{2}$ and $H^{2}(w)$. The example is a step function-such an example is necessary, as this behaviour cannot occur if the essential range of $\log (w)$ does not have large gaps (for example if the sizes of the components of the complement are bounded).

In $\S 3$ we consider the question of when, for any fixed Banach space $Y$, the continuity of a linear map $T$ from $H^{p}\left(w_{0}\right)$ to $Y$ and from $H^{p}\left(w_{1}\right)$ to $Y$ implies the continuity of $T$ from $H^{p}\left(w_{0}^{1-s} w_{1}^{s}\right)$ to $Y$. The answer is that this holds if and only if $H^{p}\left(w_{0}^{1-s} w_{1}^{s}\right)$ is contained in $H^{p}\left(w_{0}\right)+H^{p}\left(w_{1}\right)$. We show that this fails if $w_{1} / w_{0}$ has one-sided limits of zero and infinity at the same point.

In $\S 4$ we apply the positive result of $\S 1$ to a problem raised in $\left[\mathrm{DM}^{\mathrm{C}} \mathrm{C}\right]$, namely, determining which Toeplitz operators are bounded on $H^{2}\left(\left|e^{i \theta}-1\right|\right)$. This was the problem that originally inspired this paper.

We remark that there is another, nonequivalent, way of defining weighted Hardy spaces; for details, and for interpolation theorems proved for these spaces, see [ST].

Let us fix weights $w_{0}$ and $w_{1}$, a real number $p \geq 1$, and a number $s$ strictly between 0 and 1 . Any interpolation theorem between $H^{p}\left(w_{0}\right)$ and $H^{p}\left(w_{1}\right)$ will depend only on the quotient $w=w_{1} / w_{0}$, so we shall assume that $w_{0}=1$, $w_{1}=w$. For every positive real number $t$, define a pair of outer functions $u_{t}$ and $v_{t}$ by requiring that their moduli on the circle be given almost everywhere by

$$
\begin{aligned}
& \left|u_{t}\left(e^{i \theta}\right)\right|=\min \left(1, t w^{\frac{1}{p}}\left(e^{i \theta}\right)\right), \\
& \left|v_{t}\left(e^{i \theta}\right)\right|=\min \left(1,1 / t w^{\frac{1}{p}}\left(e^{i \theta}\right)\right) .
\end{aligned}
$$

The solution of the interpolation problem depends on whether one can find a positive uniform lower bound on $\left|u_{t}(z)\right|+\left|v_{t}(z)\right|$ as $z$ ranges over the unit disk $\mathbb{D}$; the following lemma asserts that the bound exists only if $\log (w)$ is in BMO.

Lemma 1.2. Let $u_{t}$ and $v_{t}$ be defined as above. There exists $c>0$ such that $\left|u_{t}(z)\right|+\left|v_{t}(z)\right|>c$ for all $z$ in $\mathbb{D}$ and all $t>0$ if and only if $\log (w)$ is in $B M O$. 
Proof. By [Ga, p. 225], $\log \left(w^{\frac{1}{p}}\right)$ fails to be in BMO if and only if, for any $M>0$, there exists $z$ in $\mathbb{D}$ such that

$$
\int_{\mathbb{T}}\left|\log \left(w^{\frac{1}{p}}\left(e^{i \theta}\right)\right)-\int_{\mathbb{T}} \log \left(w^{\frac{1}{p}}\left(e^{i \phi}\right)\right) P_{z}(\phi) d \sigma(\phi)\right| P_{z}(\theta) d \sigma(\theta)>M,
$$

where $P_{z}(\cdot)$ is the Poisson kernel for $z$. The integral in (1.3) remains unchanged if $w$ is replaced by $t w$; choose $t$ so that $\int \log \left(t w^{\frac{1}{p}}\left(e^{i \theta}\right)\right) P_{z}(\theta) d \sigma(\theta)$ is zero. Then

$$
\log \left|u_{t}(z)\right|=\int_{\left\{\theta: t w^{\frac{1}{p}}\left(e^{i \theta}\right)<1\right\}} \log \left(t w^{\frac{1}{p}}\left(e^{i \theta}\right)\right) P_{z}(\theta) d \sigma(\theta)<-\frac{M}{2}
$$

and

$$
\log \left|v_{t}(z)\right|=-\int_{\left\{\theta: t w^{\frac{1}{p}}\left(e^{i \theta}\right)>1\right\}} \log \left(t w^{\frac{1}{p}}\left(e^{i \theta}\right)\right) P_{z}(\theta) d \sigma(\theta)<-\frac{M}{2} .
$$

So (1.3) implies the existence of $t>0$ such that $\left|u_{t}(z)\right|+\left|v_{t}(z)\right|<2 e^{-M / 2}$. The converse follows from a similar argument.

How small $u$ and $v$ can be made simultaneously controls the norm of the inclusion map from $H^{p}\left(w^{s}\right)$ into $H^{p}+H^{p}(w)$ (if the former space is not contained in the latter, we shall say the norm of the inclusion is infinite).

Lemma 1.4. Let $u_{1}$ and $v_{1}$ be as in (1.1). Suppose there is a point $z$ in $\mathbb{D}$ such that $\left|u_{1}(z)\right|^{s}+\left|v_{1}(z)\right|^{1-s} \leq \varepsilon$. Then the norm of the inclusion from $H^{p}\left(w^{s}\right)$ into $H^{p}+H^{p}(w)$ is at least $(1-\varepsilon) / \varepsilon$.

Proof. Suppose the norm of the embedding is $C$, so any $f$ in $H^{p}\left(w^{s}\right)$ can be written as $g+h$, with

$$
\|g\|_{H^{p}}+\|h\|_{H^{p}(w)} \leq C\|f\|_{H^{p}\left(w^{s}\right)} .
$$

Writing

$$
F=f\left(\frac{u_{1}}{v_{1}}\right)^{s}, \quad G=g, \quad H=h\left(\frac{u_{1}}{v_{1}}\right),
$$

(1.5) is equivalent to saying that, for any $F$ in $H^{p}$ of norm one, there exist $G, H$ in $H^{p}$, with $\|G\|+\|H\| \leq C$, and satisfying

$$
\left(\frac{v_{1}}{u_{1}}\right)^{s} F=G+\left(\frac{v_{1}}{u_{1}}\right) H \text {. }
$$

Now,

$$
\begin{aligned}
|G(z)| \leq & \exp \left[\int_{\mathbb{T}} \log \left|G\left(e^{i \theta}\right)\right| P_{z}(\theta) d \sigma(\theta)\right] \\
\leq & \exp \left[\int_{\left\{\theta:\left|v_{1}\left(e^{i \theta}\right)\right|<1\right\}} \log \left(\left|v_{1}^{s}\left(e^{i \theta}\right) F\left(e^{i \theta}\right)\right|+\left|v_{1}\left(e^{i \theta}\right) H\left(e^{i \theta}\right)\right|\right) P_{z}(\theta) d \sigma(\theta)\right. \\
& \left.+\int_{\left\{\theta:\left|v_{1}\left(e^{i \theta}\right)\right|=1\right\}} \log \left|G\left(e^{i \theta}\right)\right| P_{z}(\theta) d \sigma(\theta)\right] \\
\leq & \left|v_{1}(z)\right|^{s} \exp \left[\int_{\mathbb{T}} \log \left(\left|F\left(e^{i \theta}\right)\right|+\left|H\left(e^{i \theta}\right)\right|+\left|G\left(e^{i \theta}\right)\right|\right) P_{z}(\theta) d \sigma(\theta)\right] .
\end{aligned}
$$


So if $M(z, p)$ is the maximum value an $H^{p}$ function of norm one can attain at $z,(1.6)$ asserts that

$$
|G(z)| \leq \||F|+|G|+|H||| M(z, p)\left|v_{1}(z)\right|^{s} \leq(C+1) M(z, p)\left|v_{1}(z)\right|^{s} .
$$

Similarly, $|H(z)| \leq(C+1) M(z, p)\left|u_{1}(z)\right|^{1-s}$, so

$$
|F(z)| \leq(C+1) M(z, p)\left(\left|u_{1}(z)\right|^{s}+\left|v_{1}(z)\right|^{1-s}\right) .
$$

Letting $F$ be that function that attains the value $M(z, p)$ at $z,(1.7)$ and the hypothesis of the lemma imply that $C$ is at least $(1-\varepsilon) / \varepsilon$, as desired.

We can now prove that log-convex interpolation is possible if and only if $\log (w)$ is in BMO.

Theorem 1.8. Let weights $w_{0}$ and $w_{1}$ be given, and let $0<s<1$. Then all linear operators $T$, continuous from $H^{p}\left(w_{0}\right)$ to $H^{p}\left(w_{0}\right)$ and from $H^{p}\left(w_{1}\right)$ to $H^{p}\left(w_{1}\right)$, extend to be continuous from $H^{p}\left(w_{0}^{1-s} w_{1}^{s}\right)$ to $H^{p}\left(w_{0}^{1-s} w_{1}^{s}\right)$, and satisfy $\|T\|_{H^{p}\left(w_{0}^{1-s} w_{1}^{s}\right)} \leq C\|T\|_{H^{p}\left(w_{0}\right)}^{1-s}\|T\|_{H^{p}\left(w_{1}\right)}^{s}$, for some constant $C$ independent of $T$, if and only if $\log \left(w_{1} / w_{0}\right)$ is in BMO.

Proof. Without loss of generality, assume $w_{0}=1$ and $w_{1}=w$.

To prove sufficiency, we can show that $H^{p}\left(w^{s}\right)$ is isomorphic, under the hypothesis $\log (w) \in \mathrm{BMO}$, to either the real interpolation space $K_{s, p}\left(H^{p}, H^{p}(w)\right)$ or the complex interpolation space $\left[H^{p}, H^{p}(w)\right]_{s}$. Both these assertions are true, but as the complex space is a little simpler, we shall deal with it (see [Ca] or [BL] for a definition).

Because the complex method gives $L^{p}\left(w^{s} \sigma\right)$ when applied to $L^{p}$ and $L^{p}(w \sigma)$, the complex interpolation space between $H^{p}$ and $H^{p}(w \sigma)$ must be contained in $L^{p}(w \sigma)$, and hence, since all the elements in the interpolation space are also included in $H^{p}+H^{p}\left(w^{s}\right)$, they must be analytic (in particular in the Smirnov class) and so the interpolation space is always included in $H^{p}\left(w^{s}\right)$.

To get the reverse inclusion, letting $\psi$ be the outer function with modulus $w^{-1 / p}$, one must find, for any function $\psi^{s} f$ in $H^{p}\left(w^{s}\right)$, a function $F$, continuous on $\{\zeta \in \mathbb{C}: 0 \leq \Re(\zeta) \leq 1\}$ and analytic on the interior of the strip, that takes values in $H^{p}+H^{p}\left(w^{s}\right)$, on the line $\{\zeta \in \mathbb{C}: \Re(\zeta)=0\}$ takes values in $H^{p}$, on the line $\{\zeta \in \mathbb{C}: \Re(\zeta)=1\}$ takes values in $H^{p}(w)$, vanishes at infinity, and satisfies $F(s)=\psi^{s} f$ (the values of such functions $F$ at $s$ are, by definition, the elements of the space $\left.\left[H^{p}, H^{p}(w)\right]_{s}\right)$. The appropriate function is $F(\zeta)=e^{\zeta^{2}-s^{2}} \psi^{\zeta} f$; this will work if $\Im(\log (\psi))$ is bounded, i.e., if $\log (w)$ has a bounded conjugate. Multiplying $w$ by a weight that is bounded above and below will only affect constants, so this method will work if $\log (w)$ is the sum of a bounded function and one with bounded conjugate, which is equivalent to $\log (w)$ being in BMO [Ga, p. 248].

Necessity. Assume $\log (w)$ is not in BMO, and let $u_{t}$ and $v_{t}$ be as in (1.1). Then Lemmata 1.2 and 1.4 imply that, for any constant $K$, there exists a $t$ so that the norm of the inclusion from $H^{p}\left(\left(t^{p} w\right)^{s}\right)$ into $H^{p}+H^{p}\left(t^{p} w\right)$ is larger than $K$. By duality, there is a linear functional $\Lambda$, of norm at most one on both $H^{p}$ and $H^{p}\left(t^{p} w\right)$, and norm at least $K$ on $H^{p}\left(\left(t^{p} w\right)^{s}\right)$. Now let $f$ be any nonzero function in $H^{p} \cap H^{p}\left(w^{s}\right) \cap H^{p}(w)$ (this intersection is dense in all three spaces). Then the rank-one operator $\xi \mapsto \Lambda(\xi) f$ has norm at most $\|f\|_{H^{p}}$ 
on $H^{p}$, at most $t\|f\|_{H^{p}(w)}$ on $H^{p}(w)$, and at least $K t^{s}\|f\|_{H^{p}\left(w^{s}\right)}$ on $H^{p}\left(w^{s}\right)$. Choosing $K$ larger than $C\|f\|_{H^{p}}^{1-s}\|f\|_{H^{p}(w)}^{s}\|f\|_{H^{p}\left(w^{s}\right)}^{-1}$ yields a contradiction.

Remark. The theorem is also true for $0<p<1$. The necessity argument remains unchanged; for sufficiency, one can use the real method of interpolation (see [BL]). To get an upper bound on $K(t, f)$ one solves the corona problem for $u_{t}$ and $v_{t}$ to get functions $U_{t}$ and $V_{t}$ satisfying $u_{t} U_{t}+v_{t} V_{t}=1$ and with $\left\|U_{t}\right\|$ and $\left\|V_{t}\right\|$ bounded independently of $t$ (this can be done because of Lemma 1.2). Writing $f=u_{t} U_{t} f+v_{t} V_{t} f$ yields that the quasi-norms of $f$ in $K_{s, p}\left(H^{p}, H^{p}(w)\right)$ and $H^{p}\left(w^{s}\right)$ are equivalent.

When $\log (w)$ is not in $\mathrm{BMO}$, the interpolation spaces $\left[H^{p}, H^{p}(w)\right]_{s}$ are still ones in which the polynomials are dense, evaluation at each point of the disk is continuous, and the shift is a cyclic contraction. Are the norms equivalent to ones realisable in some other way, e.g., as the $L^{p}(\mu)$-norm for some measure $\mu$ defined on the disk? In general, when is a complex interpolation space between $P^{2}\left(\nu_{0}\right)$ and $P^{2}\left(\nu_{1}\right)$ naturally isomorphic to some $P^{2}(\mu)$ (where $P^{2}(\mu)$ denotes the closure of the polynomials in $\left.L^{2}(\mu)\right)$ ?

Notice that Theorem 1.8 does not prove that, if $\log (w)$ fails to be in BMO, one cannot get $H^{p}\left(w^{s}\right)$ as an interpolation space between $H^{p}$ and $H^{p}(w)$, only that one cannot get $H^{p}\left(w^{s}\right)$ as an interpolation space of exponent $s$. The method of proof of Theorem 1.8 will yield this stronger conclusion if one can pick the function $f$ to have norm approximately 1 in $H^{p}, 1 / t$ in $H^{p}(w)$ and $1 / t^{s}$ in $H^{p}\left(w^{s}\right)$. This in turn can be done if there is a set of positive measure where $w$ is approximately $t^{-p}$, by choosing $f$ to be a function with modulus one on this set, and modulus very small on the rest of the circle. For simplicity, we state this result for one special case:

Porism 1.9. Suppose $\log (w)$ is not in BMO, and the supremum of the diameters of the bounded components of the complement of the essential range of $\log (w)$ is finite. Then there is a linear operator $T$, continuous from $H^{p}$ to $H^{p}$ and from $H^{p}(w)$ to $H^{p}(w)$, that fails to be continuous from $H^{p}\left(w^{s}\right)$ to $H^{p}\left(w^{s}\right)$.

The hypotheses are satisfied, for example, by the weight $w\left(e^{i \theta}\right)=\theta, 0 \leq \theta<$ $2 \pi$.

We note that an indication that it may be possible to interpolate but not with log-convex bounds is given by considering the simple example of $w_{0}$ being 1 on the upper semicircle, $\varepsilon$ on the lower semicircle; and $w_{1}$ being $\varepsilon$ on the upper semicircle and 1 on the lower semicircle. It is easy to check that for any linear operator $T,\|T\|_{H^{2}\left(\sqrt{w_{0} w_{1}}\right)} \leq \sqrt{2} \max \left(\|T\|_{H^{2}\left(w_{0}\right)},\|T\|_{H^{2}\left(w_{1}\right)}\right)$. It is also easy to find operators with norms approximately one on both $H^{2}\left(w_{0}\right)$ and $H^{2}\left(\sqrt{w_{0} w_{1}}\right)$ and of order $\varepsilon$ on $H^{2}\left(w_{1}\right)$. This behaviour makes the example in the next section easier to understand.

We now give an example of a weight $w$ whose logarithm is not in BMO, but for which $H^{2}(\sqrt{w})$ is an interpolation space between $H^{2}$ and $H^{2}(w)$.

Example 2.1. Parametrize the unit circle by $\theta$ going from $-\pi$ to $\pi$. For any point $z$ in the disk, let $\omega_{z}$ denote harmonic measure on the circle with respect to $z$. 
Define sets $E_{j}$ and numbers $\alpha_{j}$ inductively as follows: Let $E_{0}=\left\{e^{i \theta}: \pi \geq\right.$ $|\theta| \geq \pi / 2\}$ and $\alpha_{0}=\pi / 2$. For each $j \geq 1$, choose $\alpha_{j}>0$ so that the set $E_{j}:=\left\{e^{i \theta}: \alpha_{j-1}>|\theta| \geq \alpha_{j}\right\}$ has the property that for each $z$ in $\mathbb{D}$, either $\omega_{z}\left(\bigcup_{i=0}^{j-1} E_{i}\right) \leq 2^{-2^{j}}$ or $\omega_{z}\left(\mathbb{T} \backslash \bigcup_{i=0}^{j} E_{i}\right) \leq 2^{-2^{j}}$.

Let $w$ be the weight that is $2^{2^{j}}$ on each $E_{j}$; clearly $\log (w)$ is not in BMO. Moreover, by choosing $\alpha_{j}$ even smaller, if necessary, one can ensure that $w$ is integrable (or in any desired $L^{p}$ class).

For each $j$, let $w_{j}$ be the weight $\min \left(w, 2^{2^{j}}\right)$. Notice that

$$
\sqrt{w} \leq \sum_{i=0}^{\infty} \frac{1}{2^{2^{i-1}}} w_{i} \leq 2 \sqrt{w}
$$

So to prove that every linear operator $T$ continuous from $H^{2}$ to $H^{2}$ and from $H^{2}(w)$ to $H^{2}(w)$ is also continuous from $H^{2}(\sqrt{w})$ to itself, it is enough to prove $T$ is continuous from each $H^{2}\left(w_{j}\right)$ to itself, with a bound on the norm of $T$ independent of $j$. And this in turn will follow if we can show that each function $f$ of norm one in $H^{2}\left(w_{j}\right)$ can be written as $g+h$, with $\|g\|_{H^{2}} \leq C / 2^{2^{j-1}}$ and $\|h\|_{H^{2}(w)} \leq C$ for some universal constant $C$.

Let $t$ be $2^{-2^{j-1}}$, and let $u:=u_{t}$ and $v:=v_{t}$ be given by (1.1).

Claim. There is some $\delta>0$, independent of $j$, such that

$$
\inf \{|u(z)|+|v(z)|: z \in \mathbb{D}\} \geq \delta .
$$

Assuming the claim for the moment, one can solve the corona problem for $u$ and $v$ to get functions $U, V$ in $H^{\infty}$, with norms less than some constant $C$ depending only on $\delta$, such that $u U+v V=1$. Writing $f=u U f+v V f$ then yields the desired decomposition.

To prove the claim, pick any point $z$ in $\mathbb{D}$. Either $\omega_{z}\left(\bigcup_{i=0}^{j-1} E_{i}\right) \leq 2^{-2^{j}}$ or, for all $k \geq j, \omega_{z}\left(\bigcup_{i=0}^{k-1} E_{i}\right) \geq 2^{-2^{k}}$, so $\omega_{z}\left(E_{k}\right) \leq 2^{-2^{k-1}}$ for all $k \geq j+1$. In the former case,

$$
\log |u(z)|=\sum_{i=0}^{j-1}\left[\frac{1}{2} \log (2)\left(2^{i}-2^{j}\right) \omega_{z}\left(E_{i}\right)\right]>2^{-2^{j}}\left(-j 2^{j}\right),
$$

and this is bounded below independently of $j$. In the latter case,

$$
\log |v(z)|=\sum_{i=j+1}^{\infty}\left[\frac{1}{2} \log (2)\left(2^{j}-2^{i}\right) \omega_{z}\left(E_{i}\right)\right]>-\sum_{i=j}^{\infty} 2^{i} 2^{-2^{i}}
$$

and this too is bounded below. So the claim is proved.

Despite this behaviour, the proof of Theorem 1.8 can be sharpened to yield the following:

If $\log (w)$ is not in BMO, there exists some constant $C>0$ such that, for any $\varepsilon>0$, there exists a rank-one operator with norm less than $C$ on both $H^{2}$ and $H^{2}(w)$, greater than 1 on $H^{2}(\sqrt{w})$, and less than $\varepsilon$ on either $H^{2}$ or $H^{2}(w)$.

Suppose $X_{0}$ and $X_{1}$ are Banach spaces and $X$ is another Banach space in which $X_{0} \cap X_{1}$ is dense. Then the following proposition, whose proof is elementary, holds. 
Proposition 3.1. Let $Y$ be a Banach space. Any linear map $T$ that is continuous from $X_{0}$ to $Y$ and from $X_{1}$ to $Y$ is also continuous from $X$ to $Y$ if and only if $X$ is continuously embedded in $X_{0}+X_{1}$.

Corollary 3.2. All linear functionals continuous on $H^{p}\left(w_{0}\right)$ and $H^{p}\left(w_{1}\right)$ are also continuous on $H^{p}\left(w_{0}^{1-s} w_{1}^{s}\right)$ if and only if, for $w=w_{1} / w_{0}$, the functions $u_{1}$ and $v_{1}$ defined in (1.1) form a corona pair, i.e., satisfy $\inf _{z \in \mathbb{D}}\left|u_{1}(z)\right|+\left|v_{1}(z)\right|>0$.

Proof. Necessity follows from Lemma 1.4 and Proposition 3.1. To prove sufficiency, observe that $u_{1}^{s}$ and $v_{1}^{1-s}$ also form a corona pair, so there are $H^{\infty}$ functions $U$ and $V$ satisfying $u_{1}^{s}(z) U(z)+v_{1}^{1-s}(z) V(z)=1$. As in Example 2.1, any $f$ in $H^{p}\left(w_{0}^{1-s} w_{1}^{s}\right)$ can be written as $U u_{1}^{s} f+V v_{1}^{1-s} f$, where the first term is in $H^{p}\left(w_{0}\right)$ and the second is in $H^{p}\left(w_{1}\right)$.

Clearly $L^{p}\left(\sqrt{w_{0} w_{1}}\right) \subseteq L^{p}\left(w_{0}\right)+L^{p}\left(w_{1}\right)$; however, this need not be true if $L^{p}$ is replaced by $H^{p}$, as the following example shows.

Example 3.3. Let $w_{0}$ and $w_{1}$ be weights that satisfy

$$
\lim _{\theta \downarrow \alpha} \frac{w_{0}\left(e^{i \theta}\right)}{w_{1}\left(e^{i \theta}\right)}=0, \quad \lim _{\theta \uparrow \alpha} \frac{w_{1}\left(e^{i \theta}\right)}{w_{0}\left(e^{i \theta}\right)}=0 .
$$

Then, for any $0<s<1, H^{p}\left(w_{0}^{1-s} w_{1}^{s}\right)$ is not contained in $H^{p}\left(w_{0}\right)+H^{p}\left(w_{1}\right)$.

Proof. By a theorem of Lindelöf [Ga, p. 92], if an $H^{\infty}$ function has a one-sided limit of zero on the circle, it also has zero as a radial limit at that point; so both $u_{1}$ and $v_{1}$ will have radial limit zero at $e^{i \alpha}$ and cannot form a corona pair.

Let $m(z)=\sum_{n=0}^{\infty} a_{n} z^{n}$ be analytic. The Toeplitz operator $T_{\bar{m}}$ is the linear operator whose action on monomials is given by $T_{\bar{m}}\left(z^{k}\right)=\overline{a_{0}} z^{k}+\overline{a_{1}} z^{k-1}+$ $\cdots+\overline{a_{k}} z^{0}$. A problem that has been much studied recently is to determine, for a given weight $w$ (or in general any measure on the circle), exactly which $H^{\infty}$ functions $m$ give rise to bounded operators $T_{\bar{m}}$ on $H^{2}(w)$ (see, e.g., [Sa, LS]).

A Helson-Szegö weight $\rho$ is one that can be written as $e^{u+\tilde{v}}$, where $u$ is in $L^{\infty}$, and $\tilde{v}$ is the conjugate of a function in $L^{\infty}$ of norm less than $\pi / 2$. In [DMc ${ }^{\mathrm{C}}$, Theorem 2.3], it was proved that if $w=|p|^{2} \rho$, where $p$ is a polynomial and $\rho$ is Helson-Szegö, then an $H^{\infty}$ function $m$ gives rise to a bounded operator $T_{\bar{m}}$ on $H^{2}(w)$ if and only if $m=T_{\bar{h}} f$, for some $H^{2}$ function $f$, where $h$ is the outer function with modulus $w^{1 / 2}$ on the circle.

This theorem then solves the problem for all weights of the form $w\left(e^{i \theta}\right)=$ $\left|e^{i \theta}-1\right|^{\alpha}$, where $\alpha>-1$ is not an odd integer, because $\left|e^{i \theta}-1\right|^{\alpha}$ is HelsonSzegö for $-1<\alpha<1$; using the affirmative part of Theorem 1.8 , we can now almost solve the problem for $\alpha$ an odd integer:

Theorem 4.1. Let $w\left(e^{i \theta}\right)=\left|e^{i \theta}-1\right|^{\alpha}$, for any $\alpha>-1$. A necessary condition that the $H^{\infty}$-function $m$ give rise to a bounded Toeplitz operator $T_{\bar{m}}$ on $H^{2}(w)$ is that $m=T_{(\overline{z-1})^{\alpha / 2}} f$, for some $f$ in $H^{2}$; a sufficient condition is that $m=$ $T_{(\overline{z-1})^{(\alpha / 2)+\varepsilon}} f$, for some $\varepsilon>0$ and some $f$ in $H^{2}$.

Proof. Necessity follows from [DMc ${ }^{\mathrm{C}}$, Proposition 2.2]; sufficiency follows from the theorem quoted above, and interpolating between the spaces 
$H^{2}\left(\left|e^{i \theta}-1\right|^{\alpha+2 \varepsilon}\right)$ and $H^{2}\left(\left|e^{i \theta}-1\right|^{\alpha-2 \varepsilon}\right)$, which works because $\log \left(\left|e^{i \theta}-1\right|\right)$ is in $\mathrm{BMO}$.

How can Theorem 4.1 be improved to one in which the necessary and sufficient conditions coincide?

Toeplitz operators are a special subset of linear operators. For example, whatever weight $w$ one chooses, if $T_{\bar{m}}$ is bounded on $H^{2}(w)$ and $H^{2}\left(\frac{1}{w}\right)$, it will automatically be bounded on $H^{2}$, with norm less than or equal to the minimum of the norms on the other two spaces. Can one say under what conditions the boundedness of a Toeplitz operator on $H^{2}\left(w_{0}\right)$ and on $H^{2}\left(w_{1}\right)$ implies its boundedness on $H^{2}\left(\sqrt{w_{0} w_{1}}\right)$ ?

\section{REFERENCES}

[BL] J. Bergh and J. Löfström, Interpolation spaces, Springer-Verlag, Berlin, 1976.

[Ca] A. P. Calderón, Intermediate spaces and interpolation, the complex method, Studia Math. 24 (1964), 113-190.

[DMcC] B. M. Davis and J. E. McCarthy, Multipliers of de Branges spaces, Michigan Math. J. 38 (1991), 225-240.

[Ga] J. B. Garnett, Bounded analytic functions, Academic Press, New York, 1981.

[LS] B. A. Lotto and D. Sarason, Multiplicative structure of de Branges's spaces (to appear).

[Ri] M. Riesz, Sur les maxima des formes bilinéares et sur les fonctionelles linéaires, Acta Math. 29 (1926), 465-497.

[Sa] D. Sarason, Doubly shift-invariant spaces in $H^{2}$, J. Operator Theory 16 (1986), 75-97.

[St] E. M. Stein, Interpolation of linear operators, Trans. Amer. Math. Soc. 83 (1956), 482-492.

[ST] J. Strömberg and A. Torchinsky, Weighted Hardy spaces, Springer-Verlag, Berlin, 1989.

Department of Mathematics, Technion-Israel Institute of Technology, Haifa 32000, ISRAEL

Department of Mathematics, Indiana University, Bloomington, Indiana 47405

Department of Mathematics, California Institute of Technology, Pasadena, CaliFORNIA 91125 